Research Paper

\title{
TOFA induces cell cycle arrest and apoptosis in ACHN and 786-O cells through inhibiting PI3K/Akt/mTOR pathway
}

\author{
Dejiao He, Xuan Sun, Hongxia Yang, Xiaoli Li, Dingping Yang ${ }^{凶}$ \\ Department of Nephrology, Renmin Hospital of Wuhan University, 99 Zhang Zhidong Rd ,Wuhan, 430060, China \\ $\triangle$ Corresponding author: Dingping Yang (Ph.D, Professor, Director), Department of Nephrology, Renmin Hospital of Wuhan University, 99 Zhang Zhidong \\ Rd,Wuhan, 430060, China. Email: yangdingping163@163.com \\ (C) Ivyspring International Publisher. This is an open access article distributed under the terms of the Creative Commons Attribution (CC BY-NC) license \\ (https://creativecommons.org/licenses/by-nc/4.0/). See http://ivyspring.com/terms for full terms and conditions.
}

Received: 2018.03.29; Accepted: 2018.06.02; Published: 2018.06.23

\begin{abstract}
Cancer cells usually have a high requirement for fatty acids in order to meet the rapid proliferation and metabolism. Acetyl-CoA carboxylase- $\alpha$ (ACCA) catalyzes the carboxylation of acetyl-CoA to malonyl-CoA and has been a rate-limiting enzyme in the synthesis of long chain fatty acid and cellular energy storage. 5-tetradecyloxy-2-furoic acid (TOFA) is well known as an allosteric inhibitor of ACCA. In this study, we examined the functions of TOFA in human renal cell carcinoma (RCC) cell lines ACHN and 786-O. According to the results, TOFA inhibited $\mathrm{ACHN}$ and 786-O cell growth in a concentration and time dependent manner. The IC50 values of ACHN and 786-O cells were 6.06 and $5.36 \mu \mathrm{g} / \mathrm{ml}$ by the treatment of TOFA for $48 \mathrm{~h}$, respectively. Flow cytometry analysis showed that TOFA markedly arrest cell cycle at G2/M phase and lead to cell apoptosis. In addition, Western blot results revealed that TOFA decreased the phosphorylation of proteinkinaseB(Akt), Mammalian target of rapamycin (mTOR) and p70 ribosomal protein S6 kinase (p70S6K). What's more, specific phosphoinositide 3-kinases (PI3K) phosphorylation inhibitor LY294002 potentiated TOFA anti-cancer activity. These results suggested that TOFA induces growth restraint and apoptosis via inhibiting the PI3K/Akt/mTOR pathway and TOFA may be a novel therapeutic strategy for RCC treatment.
\end{abstract}

Key words: TOFA; Human renal cell carcinoma cell lines; Cell cycle arrest, Cell apoptosis; PI3K/Akt/mTOR

\section{Introduction}

Renal cell carcinoma (RCC) was regarded as the most lethal urological tumor [1]. The incidence and mortality rate of RCC are continuously rising at a rate of $2-3 \%$ per-decade [2]. RCC poses a threat to public healthy due to the 5-year relative survival rates at diagnosis is still poor and less than $10 \%$ of patients survive over 5 years $[3,4]$. Therefore, studying the underlying mechanisms and molecular basis of RCC is an essential prerequisite to develop more effective therapies nowadays.

The acetyl-CoA carboxylase (ACC) is at the junction of lipids synthesis and oxidative metabolic pathways. Two ACC isoforms, ACCA (ACC- $a$ ) and ACCB (ACC- $\beta$ ), have been identified in mammalians, which are encoded by different genes [5]. Acetyl-CoA-carboxylase-a (ACCA), located in cell cytoplasm, is a key rate-limiting enzyme in the process of fatty acid synthesis, while ACCB controls fatty acid oxidation progression. ACCA catalyzes the reaction of transforming acetyl-CoA to malonyl CoA, which is the initiating process of long-chain fatty acids biosynthesis. ACCA activity is strictly controlled by a metabolite-mediated allosteric mechanism as well as the transcriptional and posttranslational levels $[6,7]$.

Interestingly, ACCA is upregulated in many kinds of human cancers, such as breast and liver carcinoma, and likely contributes to promote lipogenesis and meet the need for rapid growth and proliferation [8]. The inhibitors or small interfering RNA of ACCA can block fatty acid synthesis, induced cell cycle arresting and cell growth inhibition in many types of human cancer cells, such as prostate cancer 
[9] and non-small-cell lung cancer [10]. These findings suggest that the ACCA is essential to cell proliferation and apoptosis, which may be a novel therapeutic strategy for cancer treatment.

5-Tetradecyloxy-2-furoic acid (TOFA) is a cell-permeable small molecule and also an allosteric inhibitor of ACCA. TOFA can block the synthesis of fatty acids, thus restraining the synthesis of phosphatidylcholine, which involved in the generation of cell membranes [11]. According to previous reports, TOFA suppressed proliferation and induced apoptosis in the colon cancer cell lines HCT-8 and HCT-15 [12], the prostate cancer cell line LNCAP [13] and ovarian cancer cell line COC1 [14]. However, the possible effects and mechanisms of TOFA on RCC cell lines are still not elucidated. Therefore, we investigated the roles of TOFA, acted as an ACCA inhibitor, in proliferation, cell cycle progression and apoptosis of RCC cell lines ACHN and 786-O. With original attention paid to explore the potential mechanism in the clinical management of RCC, the $\mathrm{PI} 3 \mathrm{~K} / \mathrm{Akt} / \mathrm{mTOR}$ signaling pathway mediating the effect of TOFA on the RCC cell lines ACHN and 786-O was further examined.

\section{Materials and methods}

\section{Reagents and antibodies}

TOFA was obtained from SANTA CRUZ (California, USA), Fetal bovine serum (FBS), DMEM medium and penicillin/streptomycin were obtained from Hyclone (Logan, UT, USA). 3-(4,5-dimethylthiazol-2-yl)-2,5-diphenyl-tetrazolium bromide (MTT) Annexin V-FITC/PI Apoptosis Detection Kit and Cell Cycle Assay Kit were purchased from Beyotime (Jiangsu, China). Bovine serum albumin (BSA), Dimethyl sulfoxide (DMSO), Ribonuclease (RNase A) and LY294002 were purchased from Sigma-Aldrich (St.Louis,MO,USA). RIPA Lysis Buffer, Pierce ${ }^{\mathrm{TM}}$ BCA protein assay kit, protease inhibitor cocktail, the polyvinylidene difluoride (PVDF) membrane and Super Signal West Pico Chemiluminescent Substrate detection kit was purchased from Thermo Fisher Scientific (Waltham, MA, United States). The antibodies such as p21Cip1/Waf1, CDK1, Cyclin B1, Bax, Bcl-2, Cleaved caspase 3, p-AKT (Ser473), p-mTOR (Ser2448), p-p70S6K (Ser371) and GAPDH were obtained from Cell Signaling Technology Inc. (Beverly, MA, USA).

\section{Cell culture and Cell viability assay}

ACHN and 786-O cells were purchased from Cobioer Biosciences (Nanjing, China) and cultured in DMEM medium supplemented with $10 \%$ FBS in a humidified atmosphere with $37^{\circ} \mathrm{C}$ and $5 \% \mathrm{CO}_{2}$. The stock solution of TOFA was $10 \mathrm{mg} / \mathrm{ml}$ dissolved in
DMSO. TOFA was incubated with the cells $\left(4.5 \times 10^{3}\right.$ cells/well) at various working concentrations $(0,2,4$, $6,8,10 \mu \mathrm{g} / \mathrm{ml}$ ) in 96-well plates. Viable cells were measured by MTT at an indicated time. Following $3 \mathrm{~h}$ of incubation, $100 \mu \mathrm{l} /$ well of DMSO were used to dissolve the MTT formazan crystal. The value of each well was detected on Multisken Spectrum microplate reader at $490 \mathrm{~nm}$ (Thermo Fisher Scientific).

\section{siRNA treatment}

ACCA siRNA (5'-TACAAGGGATACAGGTATT TA-3') and control siRNA (5'-CAACAAGATGAAGA GCACCAA-3') used in this study were designed and synthesized by GenePharma (Shanghai, China). In our study, the RCC cells were transfected using Lipofectamine 2000 (Invitrogene) according to manufacturer's instructions. After transfection for 24 $\mathrm{h}$, the cells were re-plated on 96-well plates and 35-mm culture dishes for indicated days to examine the cell viability and siRNA transfection efficiency.

\section{Cell cycle arrest and apoptosis analysis}

ACHN and 786-O cells were seeded at a density of $2 \times 10^{5}$ cells/well and then treated with TOFA ( 6 or 8 $\mu \mathrm{g} / \mathrm{ml})$. After $48 \mathrm{~h}$, cells were fixed in $70 \%$ ethyl alcohol for $2 \mathrm{~h}$ at $4^{\circ} \mathrm{C}$. Then cells were stained with dye mixture contain RNase and PI for $30 \mathrm{~min}$ in the dark at room temperature in order to analyze the cell cycle. For the apoptosis analysis, Annexin V-FITC/PI Apoptosis Detection Kit was used to stain the cells following the instructions. Both the cell cycle and apoptotic cells were then measured by BD FACS Calibur $^{\mathrm{TM}}$ (BD Biosciences).

\section{Western blot analysis}

The cells were harvested with RIPA lysis buffer and centrifuged for proteins. Pierce ${ }^{\mathrm{TM}}$ BCA protein assay kit was used to measure the Protein concentrations. Then $30 \mu \mathrm{g}$ protein was added to electrophoresis on 10\% SDS-polyacrylamide gel and transferred onto PVDF membranes. After blocked in $5 \%$ nonfat milk containing $0.1 \%$ Tween-20, the membranes were then incubated with primary antibodies, dissolved by $5 \%$ BSA, overnight at $4^{\circ} \mathrm{C}$. After incubated with horseradish peroxidaseconjugated secondary antibodies, the membranes were processed with the Super Signal West Pico Chemiluminescent Substrate Detection Kit following the instructions. Chemiluminescent results were detected by AmershamTM Imager 600 System (GE Healthcare Bio-Sciences, Pittsburgh, PA, United States).

\section{Statistical analysis}

For statistical analysis, all data was calculated by SPSS 18.0 software (Chicago, IL, USA) and expressed 
as mean \pm standard deviation. Differences among groups were tested by Student's T-test or one-way analysis of variance. $\mathrm{P}<0.05$ was considered statistical significance.

\section{Results}

\section{TOFA suppresses RCC cell proliferation}

In this study, we investigated the effect of TOFA on RCC cell lines ACHN and 786-O. The laddered treated cell exposed to various concentrations of TOFA $(2-10 \mu \mathrm{g} / \mathrm{ml})$ was assessed by MTT assay at indicate times. The results revealed that TOFA showed strong cytotoxicity for ACHN and 786-O cells exposed to TOFA applied for $48 \mathrm{~h}$, with an IC50 at approximately 6.06 and $5.36 \mu \mathrm{g} / \mathrm{ml}$, respectively (Figure 1). Cell proliferation analysis indicated that PI3K/Akt/mTOR pathway might involve in the inhibition effect of TOFA on ACHN and 786-O cells.

\section{ACCA siRNA decreases RCC cell proliferation}

As TOFA is an inhibitor of ACCA, we then used ACCA siRNA treatment as another way of ACCA suppression and also investigated the effect on cell growth by MTT. As described in Figure 2A and 2B, the relative expression of ACCA significantly reduced in ACHN and 786-O cells $\left({ }^{*} p<0.05,{ }^{* *} p<0.01\right.$ and *** $p<0.001$ ), respectively, when treated with $80 \mathrm{nM}$ and $100 \mathrm{nM}$ ACCA siRNA. MTT analysis in Figure 2C and $2 \mathrm{D}$ showed that suppression of ACCA by siRNA remarkably restrain the growth of $\mathrm{ACHN}$ and 786-O cells. Consistent with the change showed in Figure 1, those results presented here support that TOFA has the same effect of ACCA on the inhibition effect of RCC cell proliferation.

\section{The PI3K/Akt/mTOR pathway mediates the TOFA inhibition of ACHN and 786-O cells proliferation}

We previously determined that TOFA led to inhibiting the proliferation of ACHN and 786-O cells.

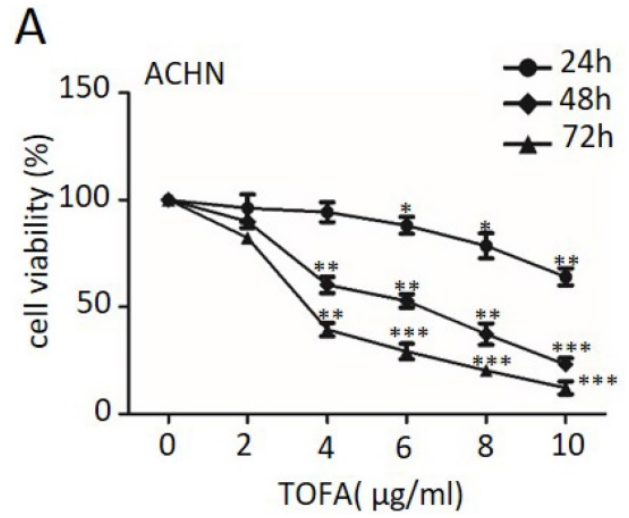

However, the underlying mechanisms of this phenomenon remained unclear. Accumulating evidence indicates the PI3K/Akt pathway plays a pivotal role in regulating the proliferation $[13,14]$. Thus, we attempted to investigate the roles of $\mathrm{PI} 3 \mathrm{~K} / \mathrm{Akt} / \mathrm{mTOR}$ pathway involving in TOFA regulation of $\mathrm{ACHN}$ and $786-\mathrm{O}$ cells.

Then we firstly treated the ACHN and 786-O cells with LY294002 (LY, $10 \mu \mathrm{M})$, the inhibitor of PI3K, for 2 hour to examine the cell viability by MTT. The outcomes in Figure $3 \mathrm{~A}$ and $3 \mathrm{~B}$ demonstrated that exposure with LY294002 alone slightly but notably decreased the cell viability in ACHN and 786-O cells $\left({ }^{*} \mathrm{p}<0.05\right)$. However, when we cast $6 \mu \mathrm{g} / \mathrm{ml}$ TOFA after pretreated with $10 \mu \mathrm{M}$ LY294002 into ACHN cells, the cell viability was remarkably concomitant decrease from $54.66 \%$ (TOFA alone) to $34.37 \%$ (TOFA+LY294002) (Figure 3A, ${ }^{* *} \mathrm{p}<0.01$ ) and the $786-\mathrm{O}$ cell viability was from $47.28 \%$ (TOFA alone) to $26.56 \%$ (TOFA+LY294002) (Figure 3B, ${ }^{* *} p<0.01$ ). These results suggested that the pathways of PI3K/Akt/mTOR might contribute the inhibition effect of TOFA on ACHN and 786-O cells.

Since incubated with TOFA would reduce the proliferation and was even more pronounced with 10 $\mu$ M LY294002, we examined the underlying proteins including the phosphorylation of Akt (Ser473), mTOR (Ser2448) and p70S6K (Ser371) by western blot analysis in Figure 3C. Specifically, our statistical results in Figure 3D demonstrated that 6 or $8 \mu \mathrm{g} / \mathrm{ml}$ TOFA alone reduces the level of p-Akt $(84.9 \%, 71.1 \%)$ in a dose-dependent manner in ACHN cells. As phosphorylated Akt activates mTOR and its downstream target p70S6K, we also observed the expression of p-mTOR $(78.3 \%, 48.1 \%)$ and p-p70S6K $(76.1 \%, 54.1 \%)$ according to the expectation. Besides, pre-treatment with LY294002 was able to remarkably aggravate this drop (p-Akt: 63.0\%, 50.7\%; p-mTOR: $39.9 \%$, 21.1\%; p-p70S6K: 33.1\%, 16.6\%; *p<0.05, $\left.{ }^{* *} \mathrm{p}<0.01\right)$ in ACHN cells. A significant dose- and LY-

B

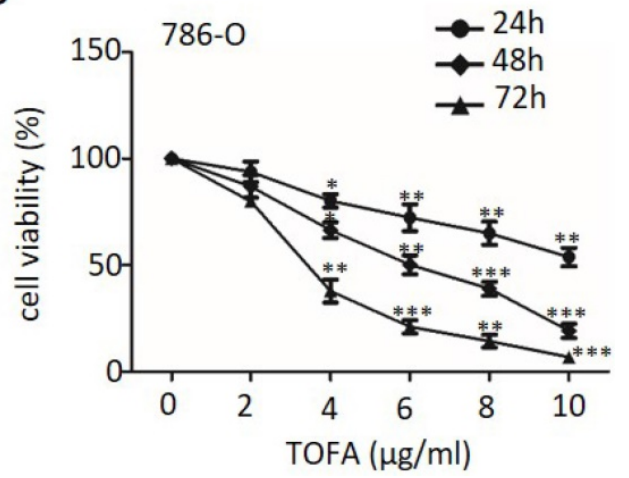

Figure 1. TOFA suppresses RCC cell proliferation. (A, B) ACHN (A) and 786-O (B) cells were treated with TOFA (DMSO, 2, 4, 6, 8 and $10 \mu \mathrm{g} / \mathrm{ml}$ ) for 24, 48, 72 h. Cell viability assessed by MTT analysis. The data was reported by mean \pm SD from three experiments. ${ }^{*} p<0.05, * *_{p}<0.01, * * * *^{*}<0.001$. 
dependent decrease of p-Akt, p-mTOR and p-p70S6K phosphorylation was also surveyed in 786-O cells (Figure $3 \mathrm{E},{ }^{*} \mathrm{p}<0.05,{ }^{* *} \mathrm{p}<0.01$ ). The findings of Figure 3 suggested that the effect of TOFA on antiproliferation propertis in ACHN and 786-O cells may be mediated by PI3K/Akt/mTOR pathway.

\section{The pathways of PI3K/Akt/mTOR is involved in TOFA-induced cell cycle arrest at G2/M phase}

Next FACS analysis was used to survey the impact of TOFA on ACHN and 786-O cells cycle arrest since proliferation of ACHN and 786-O cells was down-regulated by TOFA. Then FACS analysis and the Column Charts in Figure $4 \mathrm{~A}$ and $4 \mathrm{C}$ revealed the cell distribution at G2/M phase was a dose-dependent up-regulation from $100 \% \pm 12.8$ up to $145.7 \% \pm 10.7$ and $173.1 \% \pm 6.7$ after exposed with 6 $\mu \mathrm{g} / \mathrm{ml}$ and $8 \mu \mathrm{g} / \mathrm{ml}$ TOFA in ACHN cells, respectively $\quad\left({ }^{*} \mathrm{p}<0.05\right)$. Correspondingly, the percentage of $786-\mathrm{O}$ cells in the $\mathrm{G} 2 / \mathrm{M}$ phase was increased up to $144.0 \% \pm 15.3$ and $184.2 \% \pm 7.0$ (Figure $4 \mathrm{~B}$ and $\left.4 \mathrm{D},{ }^{* *} \mathrm{p}<0.01\right)$.

To further investigate the mechanism underlying cell cycle arrest, Western blot analysis was performed to value the expression of key cell cycle regulator including p21Cip1/Waf1, CDK1, cyclin B1. As the expression level shown in Figure 4E and $4 \mathrm{~F}$, there were significant changes in p21Cip1/Waf1 levels after

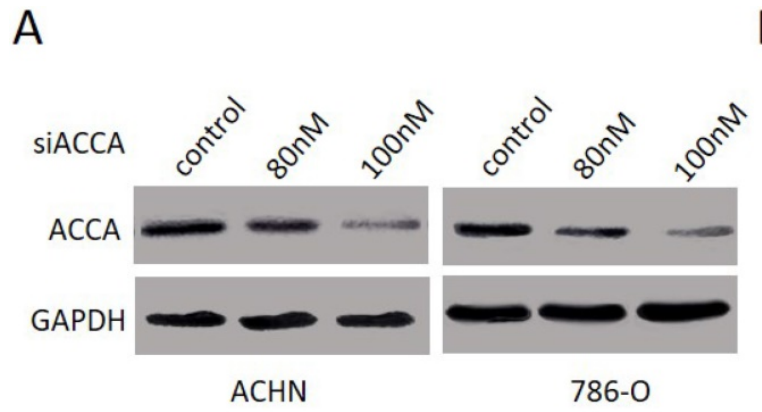

C

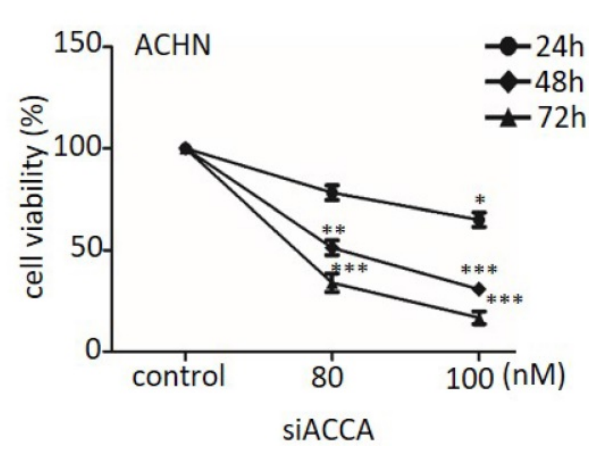

incubated with $6 \mu \mathrm{g} / \mathrm{ml}$ TOFA(4.6 fold, 2.5 fold), and even more prominent after connected with $10 \mu \mathrm{M}$ LY294002 (5.6 fold, 3.9 fold) in ACHN and 786-O cells, respectively. Furthermore, compared with control $(1.00 \pm 0.06)$, the expression of cyclin B1 and CDK1 were decrease from $0.76 \pm 0.08$ and $0.51 \pm 0.08$ (TOFA alone) to $0.50 \pm 0.03$ and $0.30 \pm 0.07$ (LY294002 and TOFA), respectively, in ACHN cells ( $\mathrm{p}<0.05)$ and the similar trends displayed in 786-O cells. This finding encouraged us to speculate that PI3K/Akt/mTOR was required for TOFA-induced G2/M phase arrest.

\section{TOFA induces RCC apoptosis through the PI3K/Akt/mTOR pathway}

Then we get some insight into the effect of TOFA on cell apoptosis by FACS analysis after ACHN and 786-O cells dealt with Annexin-V FITC/PI examined. As shown in Figure 5A and 5B, TOFA obviously increased the late apoptosis cells of ACHN at $6 \mu \mathrm{g} / \mathrm{ml}$ (8.3 fold) and $8 \mu \mathrm{g} / \mathrm{ml}$ (12.6 fold, ** $\mathrm{p}<0.01)$. Similarly, Figure 5C and 5D showed that the apoptosis cells of 786-O were significantly up-regulated following treatment with $6 \mu \mathrm{g} / \mathrm{ml}$ (11.9 fold) and $8 \mu \mathrm{g} / \mathrm{ml}(13.0$ fold) TOFA $\left({ }^{* *} p<0.01\right)$. These consistent findings in two different cells treatment supported that TOFA induced apoptosis of ACHN and 786-O cells in a dose-dependent manner.

B
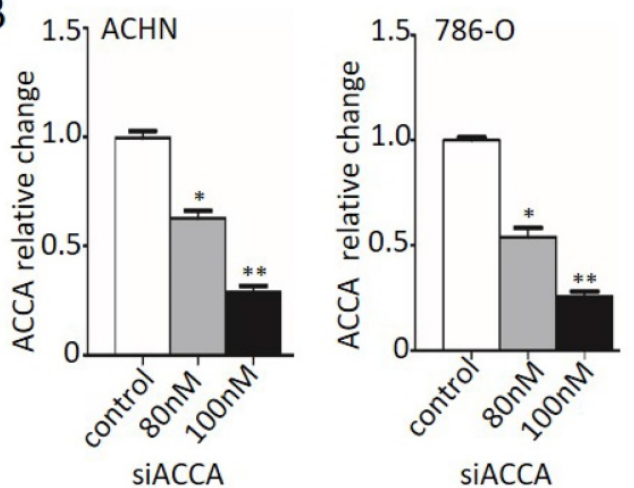

$\mathrm{D}$

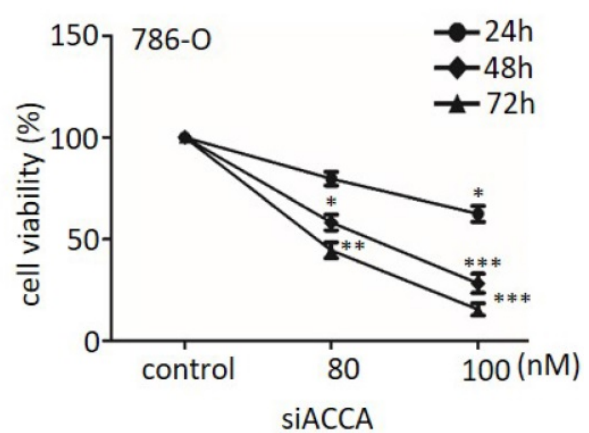

Figure 2. ACCA siRNA decreases RCC cell proliferation. (A) Western blot analysis of $A C C A(n=3)$. ACHN and $786-0$ cells were treated with 80 and 100 nM ACCA for $48 \mathrm{~h}$, and the relative expression of ACCA was analyzed by western blot. (B) Quantification of protein levels by gray value analysis. Data are expressed as the mean \pm SD. $* \mathrm{p}<0.05$; $*^{* *}<<0.01 ; * * *<0.001$. (C, D) Analysis of the effect of ACCA siRNA on the cell viability of ACHN (C) and 786-O (D) cells by MTT assay. The data was reported by mean \pm SD from three experiments. ${ }^{*} \mathrm{p}<0.05 ; * * \mathrm{p}<0.01 ; * * *<<0.001$. 
A

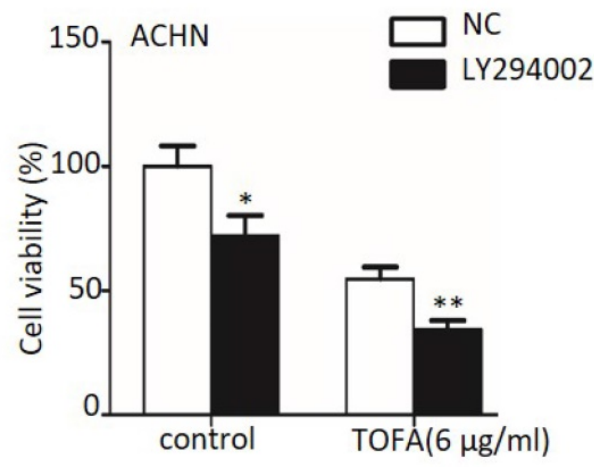

C

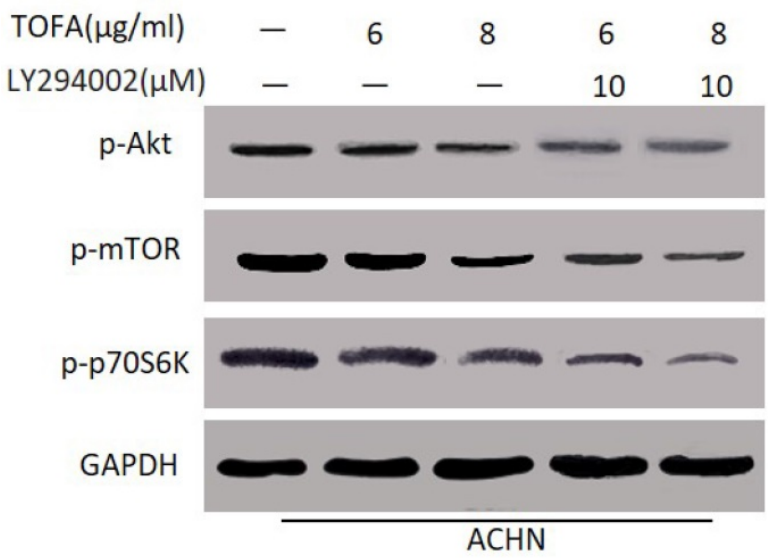

D

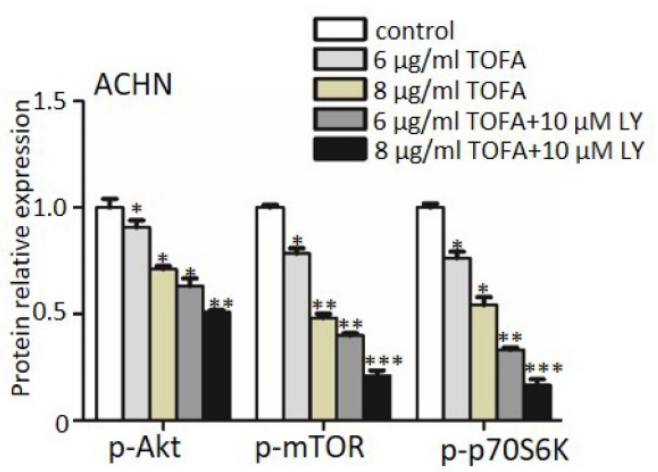

B
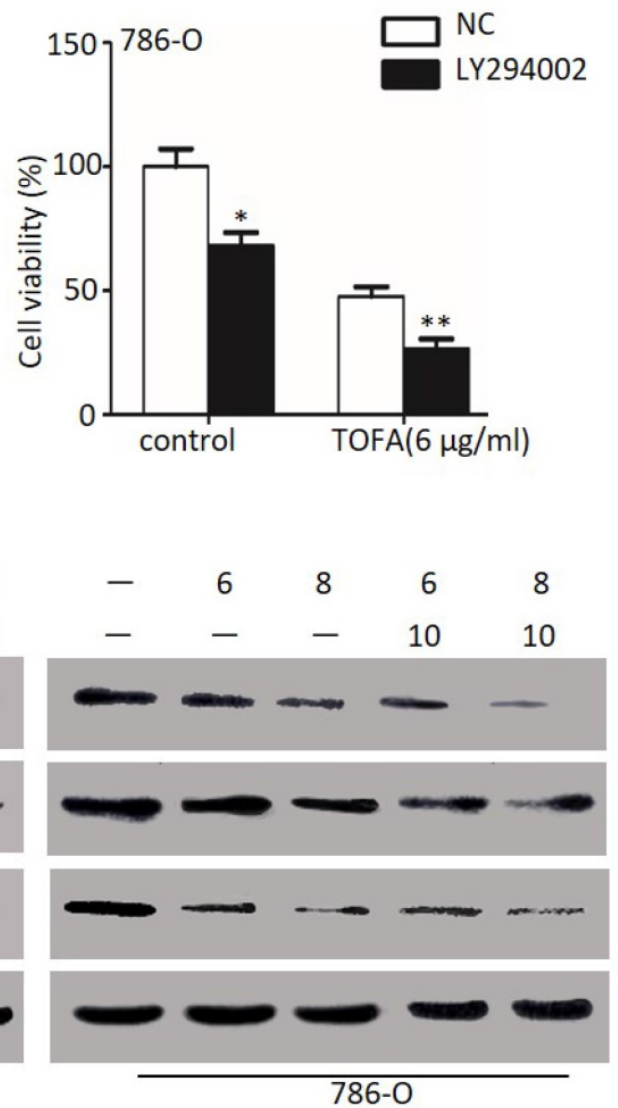

$E$

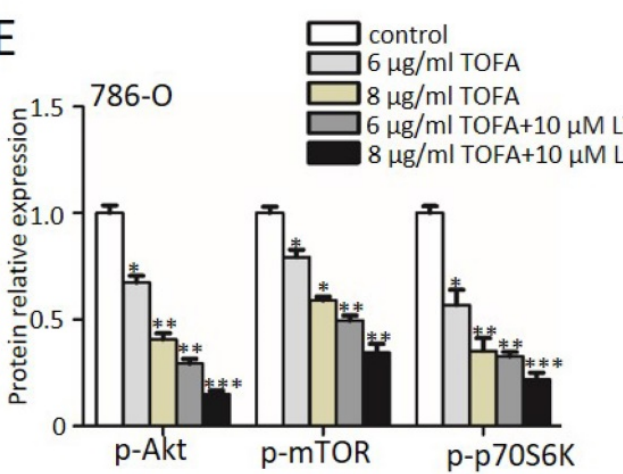

Figure 3. The PI3K/Akt/mTOR pathway mediates the TOFA inhibition of ACHN and 786-O cells proliferation. (A,B) The cell viability of $A C H N$ (A) and 786-O(B) was examined by MTT when pretreated with $10 \mu \mathrm{M}$ Ly294002 (Akt phosphorylation inhibitor) for $2 \mathrm{~h}$ before the addition of $6 \mu \mathrm{g} / \mathrm{ml}$ or $8 \mu \mathrm{g} / \mathrm{ml}$ TOFA for another 48 h. (C) Western blot analysis of the dose-dependent effects of TOFA $(6 \mu \mathrm{g} / \mathrm{ml}, 8 \mu \mathrm{g} / \mathrm{ml})$ and ly294002 $(10 \mu \mathrm{M})$ on phosphorylated and non-phosphorylated Akt, -mTOR, p70S6K. $(\mathrm{D}, \mathrm{F})$ Western blot bands of ACHN (D) and 786-O (F) corresponding to p-Akt, p-mTOR, P-p70S6K are quantified and normalized to their respective GAPDH by gray value analysis. The relative change of proteins expression compared to the control group. The data was showed as mean $\pm S D$ of three independent experiments, $* p<0.05$, $* * p<0.01$ and $* * * 0.001$.

Furthermore, the pro-apoptotic proteins Bax and cleaved caspase 3 as well as anti-apoptotic member Bcl-2 were further investigated in ACHN and 786-O cells (Figure 5E). After $48 \mathrm{~h}$ treatments with $6 \mu \mathrm{g} / \mathrm{ml}$ TOFA, the expression levels of Bax and cleaved caspase 3 were clearly increased in $\mathrm{ACHN}(2.7,2.7$ fold) and 786-O cells (4.1, 3.6 fold), while the expression was more profoundly raised when incubated with $6 \mu \mathrm{g} / \mathrm{ml}$ TOFA in presence of $10 \mu \mathrm{M}$ LY294002 (Figure 5F and 5G; ** $p<0.01$ ). However, the anti-apoptotic protein Bcl-2 led to descend to $61.4 \%$ and $45.3 \%$ in ACHN and 786-O cells following treatment with TOFA. In addition, the inhibition effect of TOFA on the expression level of anti-apoptotic protein Bcl-2 was notably enhanced after TOFA cooperated with $10 \mu \mathrm{M}$ LY294002 in ACHN (27.9\%) and 786-O cells (19.5\%), respectively. Those results supported that TOFA contributed to induce RCC apoptosis through the PI3K/Akt/mTOR pathway. 
A
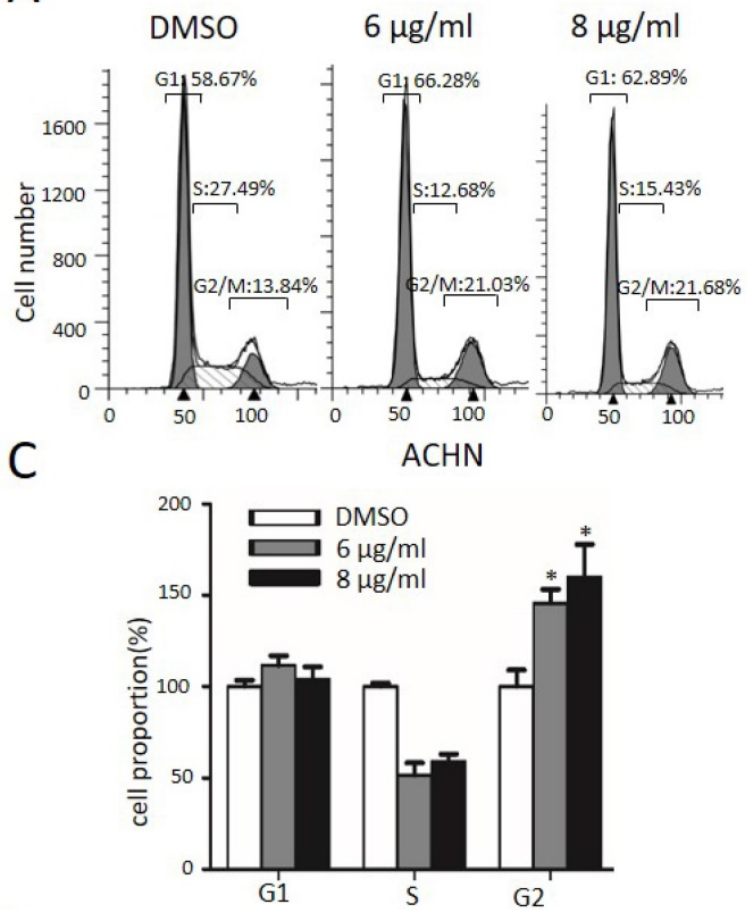

E

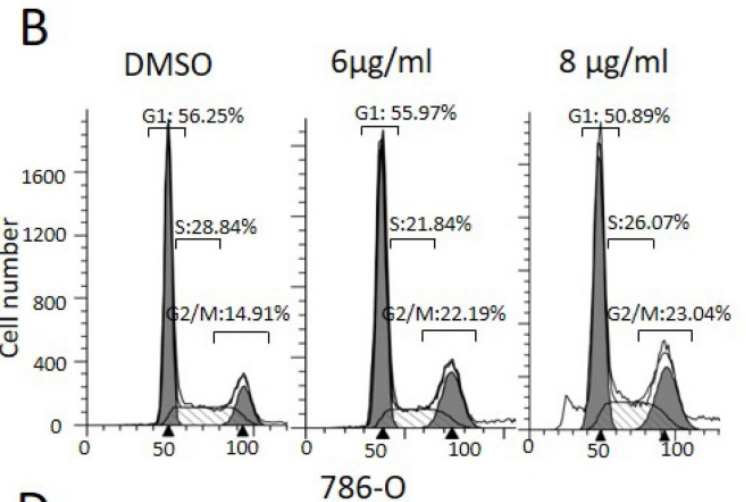

D

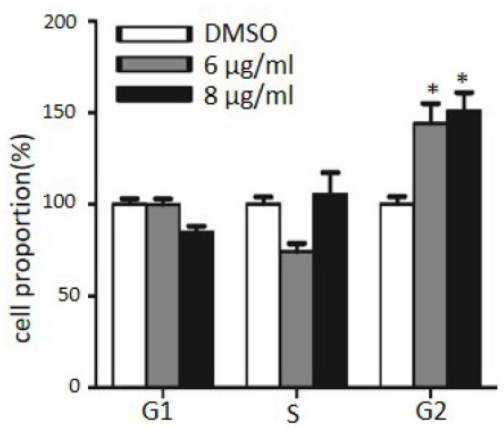

F

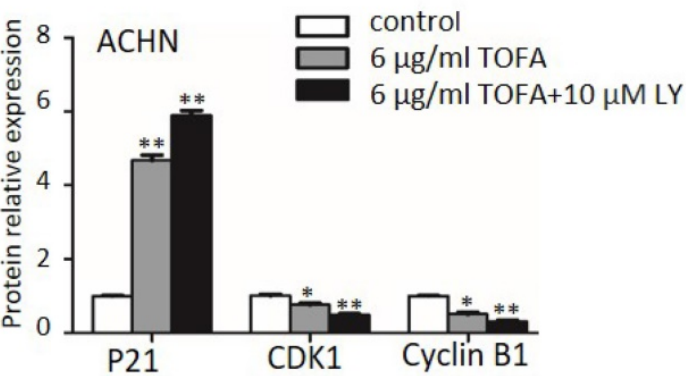

$\mathrm{H}$

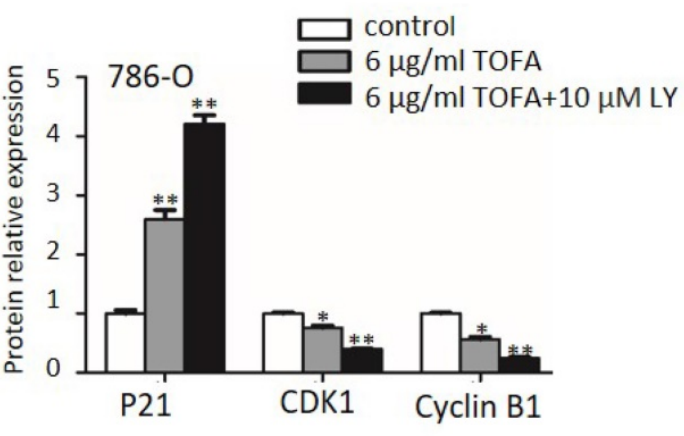

Figure 4. The pathways of PI3K/Akt/mTOR is involved in TOFA-induced cell cycle arrest at G2/M phase. (A,B) Flow cytometry analysis showed the cell cycle distribution of $A C H N(A)$ and 786-O (B) cells when treated with 4 or $6 \mu \mathrm{g} / \mathrm{ml}$ TOFA for $48 \mathrm{~h}$, respectively. (C,D) The bar graphs showed the relative cell proportion at various stages of the cell cycle when treated with TOFA in ACHN (C) and 786-O cells (D). The data was reported by mean \pm SD from three experiments. $* P<0.05$ vs the control group; **P $<0.01$ vs the control group. (E) Western blot analysis showed the expression level of $\mathrm{P} 21 \mathrm{cip} 1 /$ wafl, cdc2, cyclinB1 when treated with $6 \mu \mathrm{g} / \mathrm{ml} \mathrm{TOFA}(\mathrm{alone})$ or $6 \mu \mathrm{g} / \mathrm{ml} \mathrm{TOFA}$ and $10 \mu$ MLY294002 (LY294002 was pretreated before treatment with TOFA), GAPDH was used as the internal control. (F) The relative expression of proteins were quantified by gray value analysis. Data are displayed as the mean \pm SD. ${ }^{*} \mathrm{p}<0.05 ; * * \mathrm{p}<0.01$; $* * * \mathrm{p}<0.001$ 
A

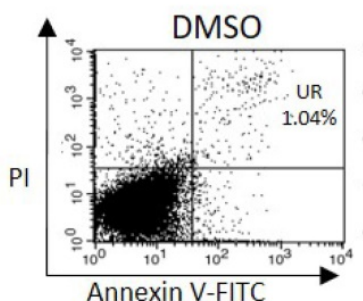

C

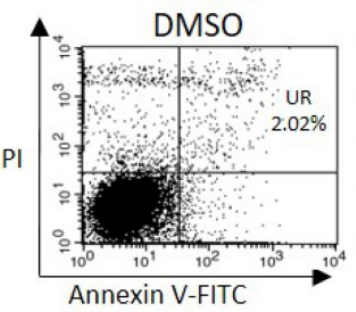

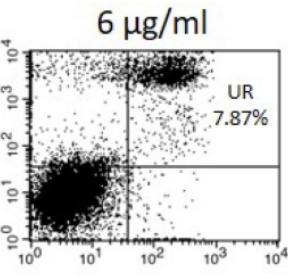
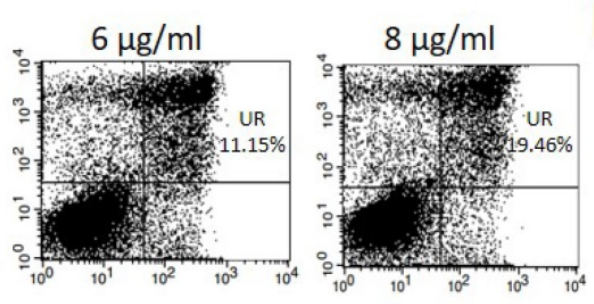

B

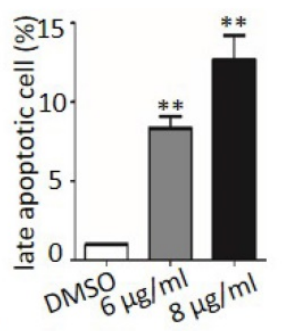

D

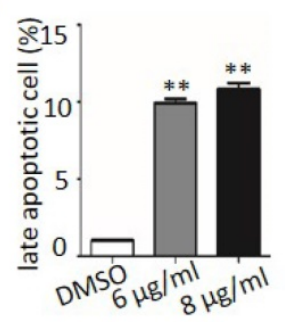

E

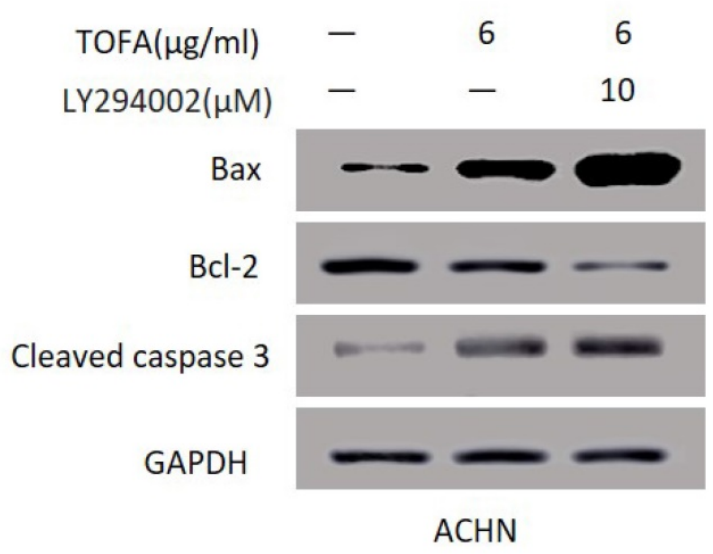

$\begin{array}{lrr}- & 6 & 6 \\ - & - & 10\end{array}$

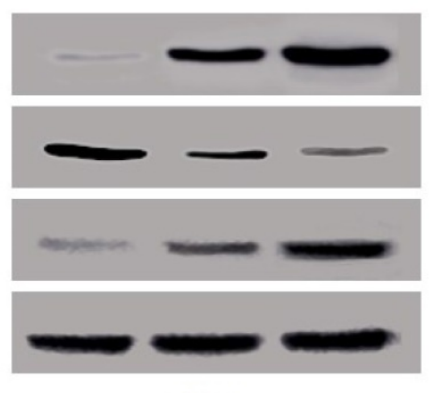

786-0

$\mathrm{F}$
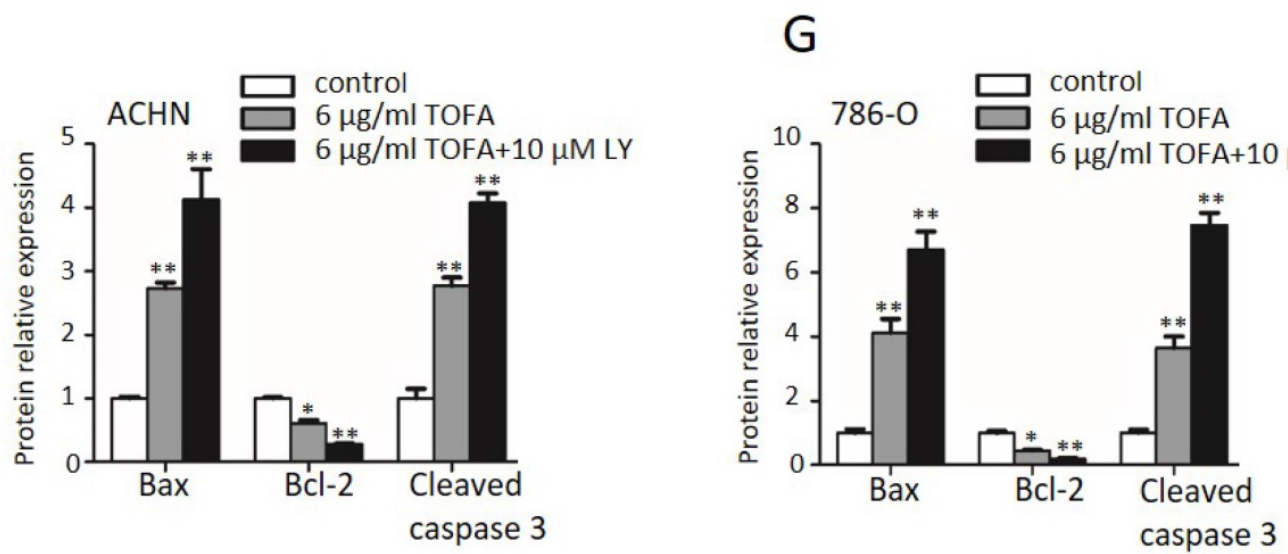

Figure 5. TOFA induces RCC apoptosis through the PI3K/Akt/mTOR pathway. (A, C) Flow cytometry showed cells in different apoptotic stages after ACHN (A) and 786-O cells (C) were exposure with $6 \mu \mathrm{g} / \mathrm{ml}$ or $8 \mu \mathrm{g} / \mathrm{ml}$ TOFA for $48 \mathrm{~h}$. (B, D) The bar graphs showed the percentage of late apoptosis cells according to A and C. Mean \pm SD represents the data from three experiments. ${ }^{* * P}<0.01$ vs the control group. $(\mathrm{E})$ The protein expression of Bax, Bcl-2, cleaved caspase 3 were showed by $\mathrm{Western}$ blot when cells were treated with $6 \mu \mathrm{g} / \mathrm{ml}$ TOFA or/and $10 \mu \mathrm{M}$ LY294002 for 48 hours. (F, G) The bar graphs represented the relative expression of Bax, Bcl-2, cleaved caspase 3 in ACHN (F) and $786-\mathrm{O}$ cells $(\mathrm{G})$ compared with control groups. Mean \pm SD reported the data from three experiments. *P<0.05 vs the control group; **P<0.01 vs the control group.

\section{Discussion}

According to reports before, fatty acid synthesis plays important role in tumor cells' survival and functional metabolism [15]. Fatty acids not only contribute as the major components of bio-membranes, but also nutrients to fulfill the requisition of rapid cell division, cell migration and signal transduction [16]. So lipogenic alterations may have effect on the progression of human tumors. In the process of fat acid synthesis, ACCA is the major rate-limiting enzyme. Upregulation of ACCA correlates with high level of palmitic acid and poor prognoses [17-19], while ACCA inhibition reduces the developing progression of tumors $[9,10]$. Thus, the fatty acid synthesis pathway may function as a novel therapeutic target of cancer. 
It has been reported that TOFA, an allosteric inhibitor of the ACCA, inhibits fatty acid synthesis thus restraining cellular growth and metabolism in many kinds of cancers. The high cytotoxic effect of TOFA on human cancer cells would suppress cell growth, induce apoptosis as well as arrest cell cycle many kinds of cancer cells [12-14]. For example, TOFA is a potent cell death inducing agent by blocking fatty acid synthesis, inhibiting expression of AR, neuropilin-1 and Mcl-1 and killing cancer cells independent of p53 status in prostate cancer cells [20].TOFA has been shown numerous anti-cancer activities and may be regarded as a potential therapeutic drug for human renal cancer.

In this study, ACHN and 786-O cells were chose to investigate the role of TOFA in vitro. We found that the growth of ACHN and 786-O cells was efficiently inhibited after TOFA treatment. ACC1 siRNA and MTT analysis in figure 2 were exerted to further identify the function of TOFA as an inhibitor of ACC1 and both suppress the proliferation of RCC cells.

A growing number of reports showed that PI3K/Akt/mTOR pathway is an important intracellular signaling pathway in regulating the cell growth, cell cycle and homeostasis. PI3K activation phosphorylates Akt, which localizes in the plasma membrane [21]. The increasing phosphorylation level of Akt activates mTOR and its downstream target p70S6K, which regulates the target substrate S6 ribosomal protein [22] and induces protein synthesis at the ribosome. In this study, we then investigated the cell viability of ACHN and 786-O by MTT when pretreated with $10 \mu \mathrm{M}$ Ly294002 (PI3K/Akt phosphorylation inhibitor) for $2 \mathrm{~h}$ before the addition of $6 \mu \mathrm{g} / \mathrm{ml}$ or $8 \mu \mathrm{g} / \mathrm{ml}$ TOFA. We found that LY294002 has a superimposed effect with TOFA, suggesting that there may be a connection between TOFA and PI3K/Akt/mTOR pathway in suppressing the proliferation. Then the functional expression of PI3K/Akt/mTOR regulated by TOFA was examined by western blot analyses. The results in Figure 5 showed that the phosphorylation of Akt, mTOR and p70S6K were dramatically inhibited by exposure with TOFA in ACHN and 786-O cells. Based on the findings of this work, we propose that $\mathrm{PI} 3 \mathrm{~K} / \mathrm{Akt} / \mathrm{mTOR}$ signaling pathways are involved in TOFA-induced cell growth inhibition in ACHN and 786-O cells.

Previous studies have demonstrated that the antiproliferation effect may associated with cell cycle arrest as well as apoptosis via suppressing of $\mathrm{PI} 3 \mathrm{~K} /$ Akt activities in various cells $[23,24]$. Then we examined the cell cycle analysis by flow cytometry after application of TOFA to the cells and checked if this change associated with the PI3K/Akt/mTOR pathway. Our results demonstrated that TOFA treatment significantly halted ACHN and 786-O cells at $\mathrm{G} 2 / \mathrm{M}$ phase. Some regulators associated with cell cycle progression altered, including CDK1, cyclin B1, p21Waf1/Cip1, were examined in our studies by western blot. As the data showed that TOFA observably augmented p21Waf1/Cip1 as well as downregulated CDK1 and cyclin B1 levels both in ACHN and 786-O cells. In addition, the combined action of $10 \mu \mathrm{M}$ LY294002 and $6 \mu \mathrm{g} / \mathrm{ml}$ TOFA treatment led to a higher expression of p21Waf1/Cip1 and lower expression of CDK1, cyclin B1 than TOFA treatment alone, suggesting that TOFA contribute to the cell cycle blocking by inhibiting PI3K/Akt/mTOR pathway.

Tumor cells escape the cytotoxic drugs relying on protection from the induction and execution of apoptosis [25]. Thus, apoptosis analysis was observed by flow cytometry after TOFA treatment in ACHN and 786-O cells. According to report, the cysteine proteases and the Bcl-2 family members are related to apoptosis events [26]. Western blot show that TOFA decreased Bcl-2 and activated caspase 3 and Bax, then induced the apoptosis response in ACHN and 786-O cells. Moreover, blocking the PI3K/Akt/mTOR pathway by LY294002 would further contribute to the expression of activated caspase 3 and Bax and induce cell apoptosis in both cells. These findings concluded that the raising of cell apoptosis activities caused by TOFA may be connected with the inhibition of PI3K/ Akt signal path in ACHN and 786-O cells.

To the best of our knowledge, we found that the ACCA inhibitor TOFA induces growth restraint, arrest cell cycle at G2/M phase and lead to apoptosis via inhibiting the PI3K/Akt/mTOR pathway. Therefore we focused on the mechanisms of anti-cancer effects of TOFA and hoped it may be a novel therapeutic strategy for RCC treatment.

\section{Competing Interests}

The authors have declared that no competing interest exists.

\section{References}

1. Ljungberg B, Campbell SC, Choi HY, et al. Corrigendum to "The Epidemiology of Renal Cell Carcinoma". Eur Urol. 2011; 60: 1317.

2. Bex A, Jonasch E, Kirkali Z, et al. Integrating surgery with targeted therapies for renal cell carcinoma: current evidence and ongoing trials. Eur Urol. 2010; 58: 819-28.

3. Xue YJ, Xiao RH, Long DZ, et al. Overexpression of FoxM1 is associated with tumor progression in patients with clear cell renal cell carcinoma. J Transl Med. 2012; 10:200.

4. Patil S, Ishill N, Deluca J, et al. Stage migration and increasing proportion of favorable-prognosis metastatic renal cell carcinoma patients: implications for clinical trial design and interpretation. Cancer. 2010; 116: 347-545.

5. Sebastian Kreuz, Corinna Schoelch, Leo Thomas, et al. Acetyl-CoA carboxylases 1 and 2 show distinct expression patterns in rats and humans and alterations in obesity and diabetes. Diabetes Metab Res Rev. 2009; 25: 577-86.

6. Witters L A, J Widmer, A N King, et al. Identification of human acetyl-CoA carboxylase isozymes in tissue and in breast cancer cells. Int J Biochem. 1994; 26: 589-94. 
7. Wakil SJ, Stoops JK, Joshi VC. Fatty acid synthesis and its regulation. Annu Rev Biochem. 1983; 52: 537-579.

8. Chajes V, Cambot M, Moreau K, et al. Acetyl-CoA carboxylase alpha is essential to breast cancer cell survival. Cancer Res. 2006; 66: 5287-5294

9. Brusselmans $\mathrm{K}$, De Schrijver E, Verhoeven G, et al. RNA interference-mediated silencing of the acetyl-CoAcarboxylase-alpha gene induces growth inhibition and apoptosis of prostate cancer cells. Cancer Res 2005; 65: 6719-6725

10. Svensson RU, Parker SJ, Eichner LJ, et al. Inhibition of acetyl-CoA carboxylase suppresses fatty acid synthesis and tumor growth of non-small-cell lung cancer in preclinical models. Nat Med. 2016; 22: 1108-1119.

11. Harwood HJ Jr. Treating the metabolic syndrome: acetyl-CoA carboxylase inhibition. Expert Opin Ther Targets. 2005; 9: 267-81.

12. Wang $\mathrm{C} 1, \mathrm{Xu} \mathrm{C}$, Sun $\mathrm{M}$, et al. Acetyl-CoA carboxylase-alpha inhibitor TOFA induces human cancer cell apoptosis. Biochem Biophys Res Commun. 2009; 385: 302-6.

13. Guseva NV, Rokhlin OW, Glover RA, et al. TOFA (5-tetradecyl-oxy-2-furoic acid) reduces fatty acid synthesis, inhibits expression of AR, neuropilin- 1 and Mcl-1 and kills prostate cancer cells independent of p53 status. Cancer Biol Ther. 2011; 12: 80-5.

14. Li S1, Qiu L, Wu B, et al. TOFA suppresses ovarian cancer cell growth in vitro and in vivo. Mol Med Rep. 2013; 8: 373-378.

15. Hopperton KE, Duncan RE, Bazinet RP, et al. Fatty acid synthase plays a role in cancer metabolism beyond providing fatty acids for phospholipid synthesis or sustaining elevations in glycolytic activity. Exp Cell Res. 2014; 320: 302-10.

16. Huang LH, Chung HY, Su HM. Docosahexaenoic acid reduces sterol regulatory element binding protein- 1 and fatty acid synthase expression and inhibits cell proliferation by inhibiting pAkt signaling in a human breast cancer MCF-7 cell line. BMC Cancer. 2017; 17: 890.

17. Kuhajda FP. Fatty-acid synthase and human cancer: new perspectives on its role in tumor biology. Nutrition. 2000; 16: 202-8.

18. Brusselmans $K$, De Schrijver E, Verhoeven G, et al. RNA interference-mediated silencing of the acetyl-CoAcarboxylase-alpha gene induces growth inhibition and apoptosis of prostate cancer cells. Cancer Res. 2005; 65: 6719-25.

19. Chajès V, Cambot M, Moreau K, et al. Acetyl-CoA carboxylase alpha is essential to breast cancer cell survival. Cancer Res. 2006; 66: 5287-94.

20. Guseva NV, Rokhlin OW, Glover RA, et al. TOFA (5-tetradecyl-oxy-2-furoic acid) reduces fatty acid synthesis, inhibits expression of AR, neuropilin-1 and Mcl-1 and kills prostate cancer cells independent of p53 status. Cancer Biology \& Therapy. 2011; 12: 80-5.

21. King, D, Yeomanson D, Bryant HE. PI3King the Lock: Targeting the PI3K/Akt/mTOR Pathway as a Novel Therapeutic Strategy in Neuroblastoma. J Pediatr Hematol Oncol. 2015; 37: 245-51.

22. Chung J, Kuo CJ, Crabtree GR, et al. Rapamycin-FKBP specifically blocks growth-dependent activation of and signaling by the $70 \mathrm{kd}$ S6 protein kinases. Cell. 1992; 69: 1227-1236

23. Nagappan A, Lee WS, Yun JW, et al. Tetraarsenic hexoxide induces G2/M arrest, apoptosis, and autophagy via PI3K/Akt suppression and p38 MAPK activation in SW620 human colon cancer cells. PLoS One. 2017; 12:e0174591.

24. Berrak Ö, Akkoç Y, Arısan ED, et al. The inhibition of PI3K and NFkB promoted curcumin-induced cell cycle arrest at $\mathrm{G} 2 / \mathrm{M}$ via altering polyamine metabolism in Bcl-2 overexpressing MCF-7 breast cancer cells. Biomed Pharmacother. 2016; 77: 150-60

25. Pan ST, Oin $Y$, Zhou ZW, et al. Plumbagin induces G2/M arrest, apoptosis, and autophagy via p38 MAPK- and PI3K/Akt/mTOR-mediated pathways in human tongue squamous cell carcinoma cells. Drug Des Devel Ther. 2015; 9:1601-26.

26. Métrailler-Ruchonnet I, Pagano A, Carnesecchi S, et al. Bcl-2 protects against hyperoxia-induced apoptosis through inhibition of the mitochondriadependent pathway. Free Radic Biol Med. 2007; 42: 1062-74. 